\title{
Reactivity and resizing of gold nanorods in presence of $\mathrm{Cu}^{2+}$
}

\author{
T S SREEPRASAD, A K SAMAL and T PRADEEP* \\ Department of Chemistry and Sophisticated Analytical Instrument Facility, Indian Institute of Technology \\ Madras, Chennai 600 036, India
}

\begin{abstract}
Due to the inherent anisotropy of the system, gold nanorods behave differently in comparison to their spherical counterparts. Reactivity of gold nanorods, in presence of cupric ions, was probed in an attempt to understand the chemistry of anisotropic particles. The reaction progresses through a series of intermediates. It can be arrested at any stage to get nanorods of desired dimension and therefore, can be used for their reshaping. The presence or absence of cetyltrimethylammonium bromide (CTAB) on the nanorod surface was found to be determining the site of initiation of the reaction. When a large concentration of CTAB is present in the system, selective etching of the tips of the nanorod occurs and when the nanorods are purified to reduce the amount of CTAB in the solution, the side faces of the nanorod also get reacted. Gold nanorods are converted to particles by further surface reconstructions in a systematic surface specific chemistry.
\end{abstract}

Keywords. Gold nanorods; anisotropic particles; $\mathrm{Cu}^{2+}$.

\section{Introduction}

Scientific interest in the nano regime lies in the fact that it is considered as a bridge between the bulk and atomic or molecular structures. The size dependent properties of nanomaterials make them interesting and technologically important (Rao et al 2000). Quantum confinement in semiconductor particles (Norberg et al 2006) and characteristic surface plasmon resonances (SPR) in certain metal particles (Smith and Corn 2003; Jin and Friedman 2005) are some interesting examples of size dependent properties. Nanomaterials by virtue of their high surface to volume ratio exhibit very different chemical reactivity and properties, compared to their bulk counterparts (Zhan et al 2002; Prakash et al 2005). An example is the reactivity of $\mathrm{Au}$ and $\mathrm{Ag}$ nanoparticles towards halocarbons, which has been studied extensively (Nair and Pradeep 2003, 2004; Nair 2006). The reactions investigated can be extended for the degradation of some common pesticides present in surface waters. We devised an economical and efficient drinking water purifier based on this reaction in collaboration with Eureka Forbes Ltd. (Nair and Pradeep 2007).

Gold nanorods are excellent anisotropic nanostructures for a number of applications due to their size-dependent optical properties (Juste et al 2005). Nanorods can be prepared through several methods such as electrochemical deposition in hard templates (Foss Jr et al 1992) and at step edges (Martin 1996), electrochemical synthesis in solution (Yu et al 1997; Chang et al 1999), photochemical synthesis (Kim et al 2002), microwave heating (Liu et al 2004) and by the seed-mediated growth (Sau and Murphy

*Author for correspondence (pradeep@iitm.ac.in)
2004). Gold nanorods are characterized by two characteristic absorption maxima in the UV-vis spectrum. The first one, called the transverse surface plasmon resonance (TSP), is located around $520 \mathrm{~nm}$. The other maximum, known as the longitudinal surface plasmon (LSP) resonance, appears at a longer wavelength. LSP is extremely sensitive to the nanorod aspect ratio (Juste et al 2005).

Nanorods have an edge over the spherical counterparts and that they can be assembled in different ways like end-to-end, side-by-side and parallel or perpendicular to the substrate. All of these are attractive for several applications such as surface-enhanced Raman spectroscopy or fluorescence sensing applications. Hexagonal patterns of gold nanorods were made by growing them on the surface of microgels and subsequently allowing the nanorod loaded microgels to self-organize. This opens up a new possibility for making patterns in high yield through solution phase methods (Rajeev Kumar et al 2007). Nanoparticle assemblies can be used as sensors. The generation of a potential difference, of the order of tens of millivolts, induced by the flow of polar liquids over an assembly of gold nanoparticles has been reported by our group (Subramaniam et al 2005). The potential generated is in transverse direction to the flow and is dependent on the nature of the flowing liquid. We proposed a simple theoretical model, based on coulomb interaction of the flowing dipole with surface charges on nanoparticles to account qualitatively for the generation of the flow induced transverse potential. This has been extended to anisotropic nanostructures such as nanorods also (Subramanian et al 2007). Modifications in the theoretical model have been done to explain the nonlinear dependence of the potential difference on flow rate.

Gold nanorod chemistry is found to be different from their spherical counterparts owing to the surface structure 
and the presence of the $\{110\}$ facet which is absent in the spherical nanoparticles. The photothermal properties and electron dynamics (Song et al 2005), catalysis (Kline et al 2005) and biological applications (Salem et al 2003) of these systems have been investigated in great detail. Our group has recently investigated the electrostatic adsorption of hemoproteins on gold nanorods. The study suggested the retention of the molecular structure after immobilization on the nanoparticle surface. The biochemical activity of these electrostatically adsorbed hemoproteins was assessed using the selective affinity of prosthetic heme moiety towards metabolic inhibitor, azide ion (Tom et al 2007).

For any particular application, whether it involves individual particles or an assembly, the monodispersity and the size are two very important quantities that should be addressed with utmost care. Since the properties of nanorods are highly dependent on the dimension, researchers are interested in devising new methodologies for preparing monodisperse materials. One strategy is to device various synthetic methodologies to prepare different sizes of rods. The second strategy is to make a monodisperse sample from a crude polydisperse sample post-synthetically (Link et al 2000; Jana et al 2002; Rodriguez-Fernandez et al 2005; Tsung et al 2006; Mohamed et al 2007). The latter has an added advantage that differently sized nanorods can be prepared from the same starting material, simply by adjusting the reaction parameters. In this paper, we describe the surface dependent chemical reactivity of gold nanorods in presence of cupric ions. Although the distribution of the particle sizes in the in-between stages of the reaction is large, this method may be developed further to yield particles of desired shape. This is a continuation of our earlier work on this problem (Sreeprasad et al 2007) and additional data are presented.

\section{Experimental}

\subsection{Synthesis of gold nanorods}

Nanorods were made as per the reported procedure which followed the well-established seed-mediated method (Sau and Murphy 2004). First, CTAB protected gold seeds were prepared by the reduction of the required quantity of gold ions by ice-cold $\mathrm{NaBH}_{4}$ in presence of CTAB. After $3 \mathrm{~h}$, a calculated amount of the seed solution was added to a growth solution containing specific amounts of CTAB, $\mathrm{HAuCl}_{4}$ and ascorbic acid. The resultant solution was kept without disturbance for $24 \mathrm{~h}$ and examined using TEM to determine the monodispersity of the sample.

The as-prepared nanorod sample $\left(R_{1}\right)$ was centrifuged at $11,100 \mathrm{rpm}$ for $15 \mathrm{~min}$. The supernatant was discarded and the residue was redispersed in an equal amount of triply distilled water. The sample was again centrifuged at $11,100 \mathrm{rpm}$. This was repeated twice, principally to remove excess CTAB and other impurities present. The sample will be referred to as $R_{2}$ in the paper hereafter.

\subsection{Reactivity study}

The as-prepared $\left(R_{1}\right)$ as well as the purified $\left(R_{2}\right)$ samples were subjected to reaction separately. The chemical reactivity of nanorods with metal chlorides like $\mathrm{CuCl}_{2}$ was studied. The nanorod solution was mixed with $\mathrm{CuCl}_{2}$ solution and the mixture was heated in a water bath maintained at $70^{\circ} \mathrm{C}$. The UV-vis spectrum and TEM of the sample were analysed every $1 \mathrm{~h}$. The reaction was done both with $R_{1}$ and $R_{2}$ as described by Sreeprasad et al (2007).

\section{Instrumental}

A Perkin-Elmer Lambda $25 \mathrm{UV}$-visible spectrophotometer was used to measure the UV-vis spectrum of the samples. Samples were drop casted on a carbon coated copper grid and dried in ambience. This was observed under a JEOL 3011, $300 \mathrm{kV}$ Transmission Electron Microscope (TEM) with an ultra high resolution (UHR) pole piece.

\section{Results and discussion}

Figure 1 shows the UV-vis spectra acquired at various stages of the reaction between $R_{1}$ and $\mathrm{CuCl}_{2}(1 \mathrm{mM})$. The position of plasmon gives us an idea about the aspect ratio of the gold nanorod. The LSP shifts linearly with aspect ratio of the nanorods (Juste et al 2005). As we can see the LSP blue shifts and decreases in intensity, while the TSP red

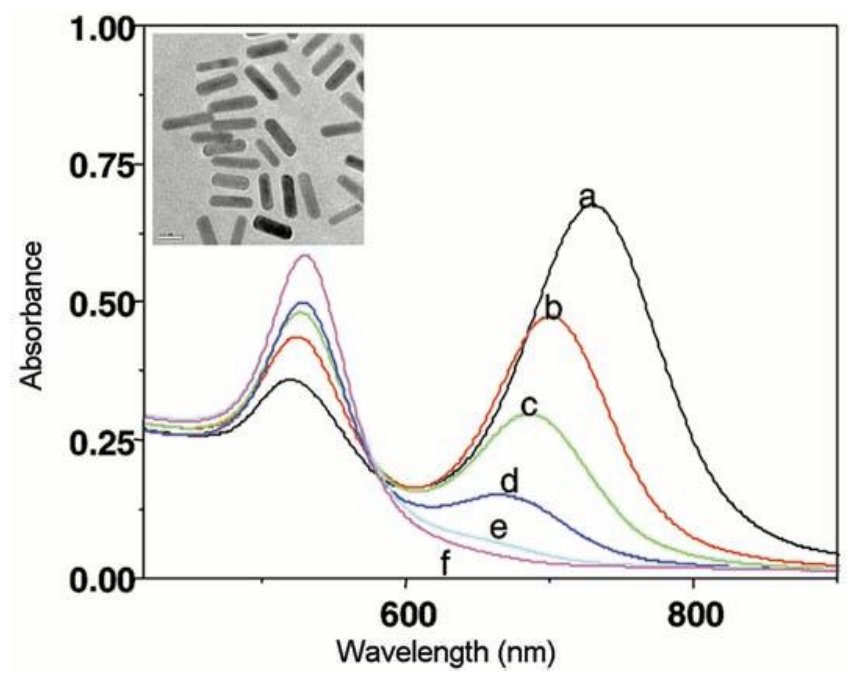

Figure 1. UV-vis spectra of the reaction between $\mathrm{Au}$ nanorods and $\mathrm{CuCl}_{2}$ as a function of time. Spectra were acquired every $1 \mathrm{~h}$ of the reaction (a-f). Trace $a$ corresponds to the parent nanorod sample and $b$ is after $1 \mathrm{~h}$ of the reaction (Inset: TEM image of pristine nanorods). 


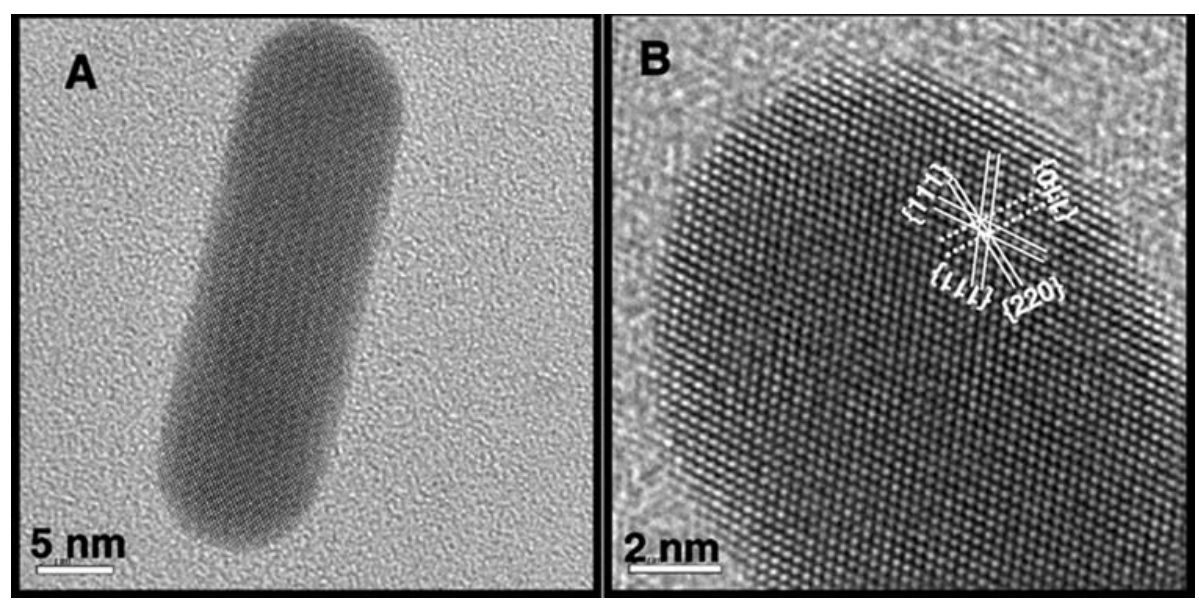

Figure 2. A. Lattice resolved image of a pristine nanorod showing the well defined tip and side devoid of any defects and $\mathbf{B}$. a high magnification image of a nanorod tip.

shifts slightly and increases in intensity during the reaction. After $3 \mathrm{~h}$ of the reaction, only a hump is shown in place of LSP and finally after $4 \mathrm{~h}$ of the reaction, only TSP remains. All these spectral changes suggest the conversion of nanorods into spherical nanoparticles. The red shift in TSP from $518 \mathrm{~nm}$ to $531 \mathrm{~nm}$ indicates that the resultant particles formed have a larger diameter compared to the parent nanorods. The inset of figure 1 shows a large area image of pristine nanorods. We can see that they are highly monodisperse with a diameter of $\sim 13 \mathrm{~nm}$ and a length of $\sim 43 \mathrm{~nm}$.

Figure 2A shows a lattice resolved image of a pristine nanorod showing well-defined tips and sides devoid of defects. Figure 2B shows a lattice resolved image of the tip of a nanorod before the reaction. All the rods seen are single crystalline and in majority of the cases examined, the rod axis is oriented along the [100] direction and the side faces are $\{110\}$. The tips are well structured with $\{111\}$ and $\{100\}$ facets (Wang et al 2000).

Figure 3 shows the TEM images of the nanorods at a few stages of the reaction of $R_{1}$. We can see the truncated structures formed which gives an indication that the reaction starts from the tip of the rods. We can also detect a gradual decrease in the length of the rods during the course of the reaction and the end product is larger spherical particles. It is clear that specific and well-defined shapes can be created. Monodispersity of the samples in the inbetween stages is not as good as the starting material or the end product. But we can see that the rods or particles produced during the reaction are similar in diameter in comparison to the parent material.

The difference in the surface energies of different planes present on the gold nanoparticle surface gives us a tool to selectively react with those planes and get the desired shape or size (Todd and Lynden-Bell 1993; Uppenbrink et al 1994). We found that when excess CTAB is present, the edge faces of the nanorod comprising mainly of $\{111\}$ or $\{100\}$ are getting reacted. But when the nanorods are purified, the capping of the $\{110\}$ face becomes defective and the defects sites act as the reaction centres. So in the purified sample, the reactions get initiated at the defect sites and the rod body composed of $\{110\}$ facet is also getting reacted. This shows that the presence or absence of the surfactant can control the reaction. This is believed to be due to the extra stability offered to the otherwise unstable $\{110\}$ facet by the binding of CTAB.

Figure 4A shows an intermediate present in the case of reaction with $R_{1}$. We can clearly see the tips are getting reacted. In $R_{2}$, the rod body is also getting reacted (figure 4B). This is because when CTAB protects the $\{110\}$ surface, which constitutes the side faces, the reaction happens at the less protected rod tip composed of $\{111\}$ and $\{100\}$. But when the CTAB protection is defective, as a result of purification, the reaction happens in the exposed $\{110\}$ surface which is having the highest surface energy among the low index planes of gold. So when CTAB is present, due to the extra stability afforded to the $\{110\}$ surface by $\mathrm{CTAB}$, the reaction deviates from path expected from surface energy calculations and in $R_{2}$ where most of the CTAB is removed, the reaction follows the expected path, i.e. according to the surface energy (Wang et al 2000). When nanoparticles are formed during the reaction, which are mainly composed of either $\{111\}$ or $\{100\}$ surfaces (Wang et al 2000), the system is stable (Todd and Lynden-Bell 1993) and no further reaction happens. In addition, the surfaces of the particles formed will be protected by CTAB in the medium.

The reaction can be quenched by removing the heating and we found that no reaction happens at room temperature. Thus, by controlling the reaction time and temperature, we can manipulate the size of the nanorods precisely. In the absence of the control, the reaction gives spherical 

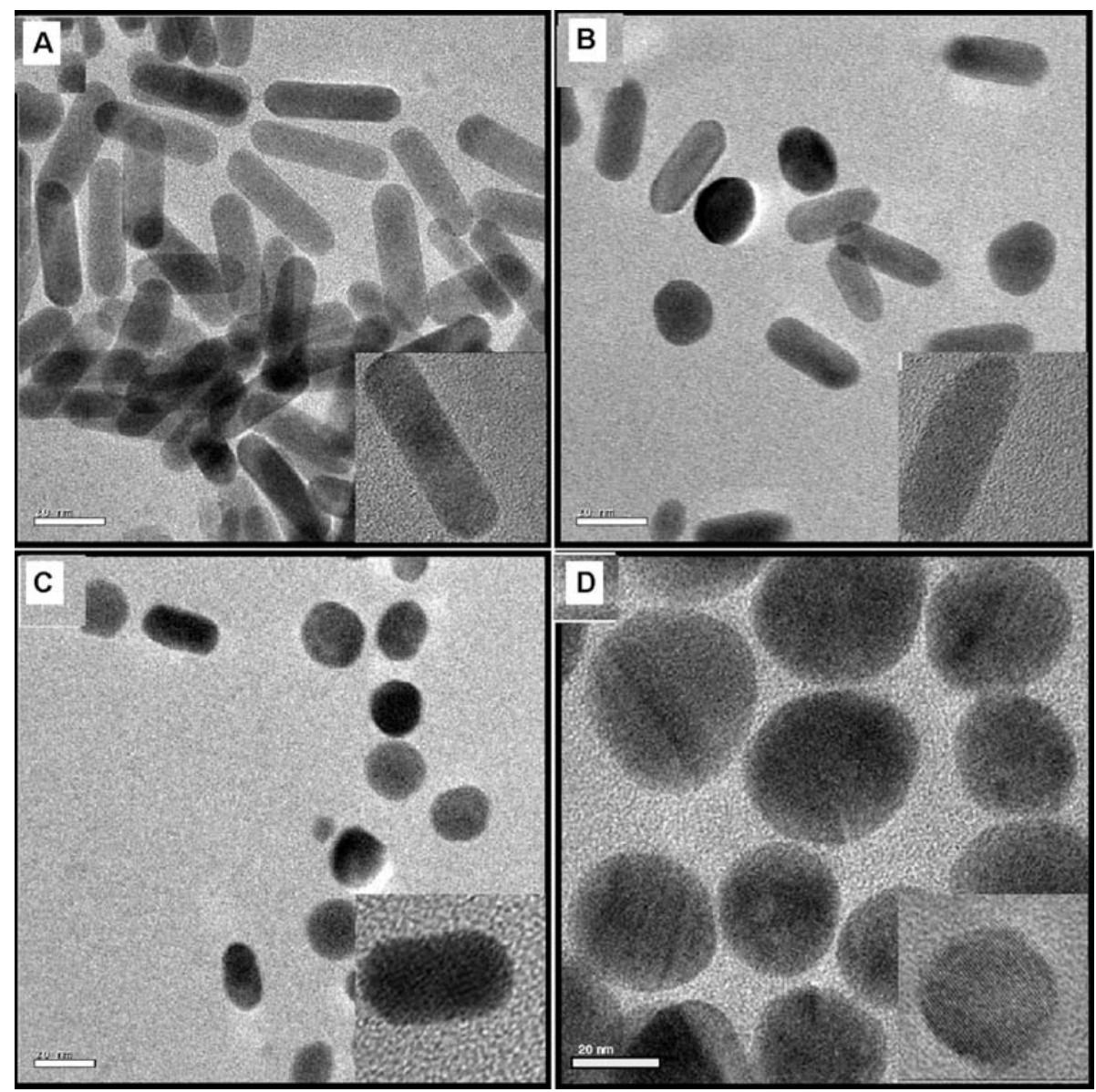

Figure 3. TEM images of $R_{1}$ during the course of transformation into particles. A. Pristine nanorods, $\mathbf{B}$. after $1 \mathrm{~h}$ of reaction, $\mathbf{C}$. after $4 \mathrm{~h}$ and $\mathbf{D}$. the final spherical particles after $6 \mathrm{~h}$ of reaction. Insets show the lattice resolved images. Scale bars in all the images are $20 \mathrm{~nm}$.

nanoparticles. The reaction product in $R_{1}$ (i.e. nanoparticles) are infinitely stable under the reaction conditions.

UV-vis and TEM data supported the conjecture that partial and selective etching happens to nanorods and the shape changes from rod to spherical particles. The reaction stops after the particles are formed. A concomitant increase in the intensity of TSP with the decrease in the intensity of the LSP shows that the conversion happens in a quantitative manner. This gives us a tool for manipulating the size and shape post-synthetically, from a single crude starting material. The reaction starts from the tip of the nanorods when they contain excess $\mathrm{CTAB}$ and when CTAB is removed, the reaction occurs on the rod body also. The surface which gets reacted is determined by the presence or absence of the protecting CTAB bilayer. The reaction showed a strong dependence on the $\mathrm{Cu}^{2+}$ concentration as well as the temperature of the reaction medium. Several control experiments were done to establish the mechanism of the reaction. We found that the purified nanorods did not show any reactivity with $\mathrm{CuCl}_{2}$ when heated. We also found that when ascorbic acid was added to $R_{2}$, a reaction similar to $R_{1}$ took place and the nanorods got reshaped to particles. No reaction occurred in Ar atmosphere both with $R_{1}$ and $R_{2}$. There are reports that $\mathrm{Cu}^{2+}$ in presence of ascorbic acid and $\mathrm{O}_{2}$ can produce hydroxyl radical (Kadiiska et al 1992; Asplund et al 2002). The reduction potential of hydroxyl radical (Koppenol and Liebman 1984) is such that it can oxidize the $\mathrm{Au}(0)$ to $\mathrm{Au}(\mathrm{I})$ which in turn results in the shortening of nanorods. This reaction starts in the more exposed $\{111\}$ plane in $R_{1}$, making the tips to get etched away. When the excess $\mathrm{CTAB}$ is removed and defects are produced in the CTAB capping, the reaction gets initiated on the thermodynamically less stable $\{110\}$ surface and the side faces get reacted. Since in $R_{2}$, ascorbic acid is not present, the reaction does not happen. In presence of $\mathrm{Na}_{2} \mathrm{SO}_{3}$, an oxygen scavenger (Carretero et al 1998), the reaction gets retarded. In presence of dimethyl sulphoxide, which acts as a hydroxyl radical scavenger (Bruck et al 2001), the reaction is retarded. All these observations made us to conclude that the reaction occurs as a result of the formation of hydroxyl radicals in presence of $\mathrm{Cu}^{2+}$ and ascorbic acid 


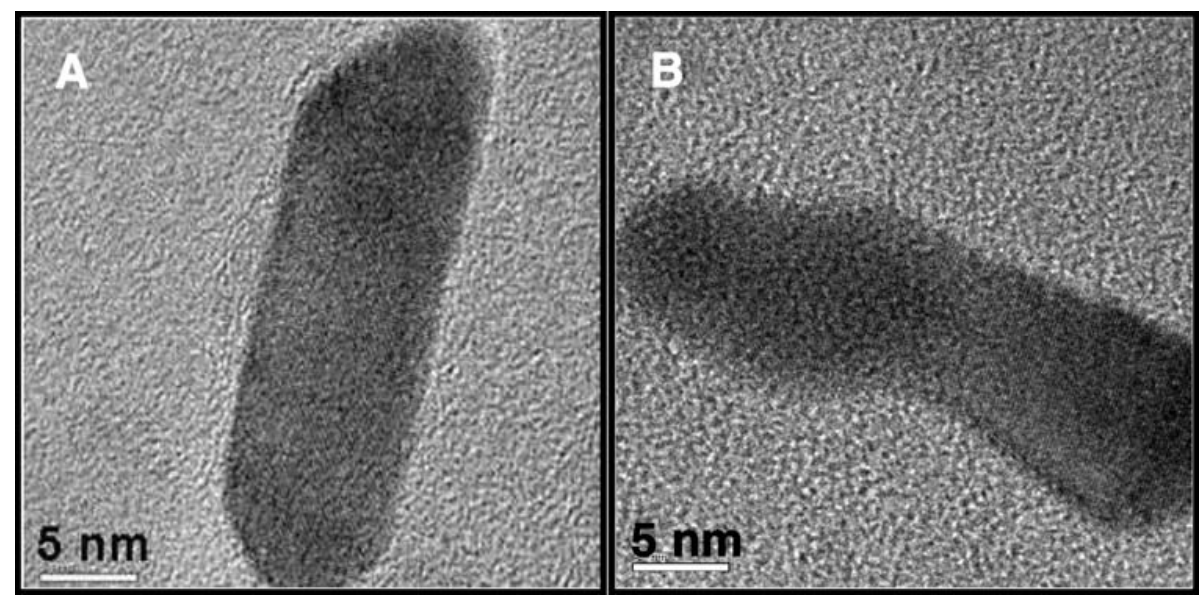

Figure 4. A. Reaction intermediate in $R_{1}$, where excess $\mathrm{CTAB}$ is present and $\mathbf{B}$. in $R_{2}$, where the excess CTAB is removed by centrifugation.

(Sreeprasad et al 2007). As the final particles formed at the end of the reaction in figure $3 \mathrm{D}$ are larger and no gold ions are detected in the solution, it is concluded that the ions etched away from the tips are reduced on the nanoparticle surface.

\section{Conclusions}

The chemistry of anisotropic nanostructures such as nanorods is similar to spherical particles in several cases, but there are also significant differences. We found that using a simple reaction with $\mathrm{CuCl}_{2}$, nanorods can be resized within a range limited by the size of the starting sample. The reaction, if extended, results in reshaping and finally yields spherical nanoparticles. In presence of excess $\mathrm{CTAB}$, reaction starts from the edge and when they are removed by centrifugation, rod body gets reacted preferentially. HRTEM and UV-vis data support the proposition. The preferential reactivity is believed to be due to the stabilizing ability of CTAB which adsorbs strongly on $\{110\}$ and gives stability to this face and the reaction happens preferentially on other facets.

\section{Acknowledgements}

We thank the DST, Government of India, for supporting our research program on nanomaterials.

\section{References}

Asplund K U M, Jansson P J, Lindqvist C and Nordström T 2002 Free Radical Res. 361271

Bruck R, Shirin H, Aeed H, Matas Z, Hochman A, Pines M and Avni Y 2001 J. Hepatol. 35457

Carretero A S, Blanco C C, Diaz B C and Gutiérrez A F 1998 Anal. Chim. Acta 361217
Chang S -S, Shih C -W, Chen C -D, Lai W -C and Wang C R C 1999 Langmuir 15701

Foss Jr C A, Hornyak G L, Stockert J A and Martin C R 1992 J. Phys. Chem. 967497

Jana N R, Gearheart L, Obare S O and Murphy C J 2002 Langmuir 18922

Jin Y and Friedman N 2005 J. Am. Chem. Soc. 12711902

Juste J P, Santos I P, Liz-Marzan L M and Mulvaney P 2005 Coord. Chem. Rev. 2491870

Kadiiska M B, Hanna P M, Hernandez L and Mason R P 1992 Mol. Pharmacol. 42723

Kim F, Song J H and Yang P 2002 J. Am. Chem. Soc. 124 14316

Kline T R, Paxton W F, Mallouk T E and Sen A 2005 Angew. Chem., Int. Ed. 44744

Koppenol W H and Liebman J F 1984 J. Phys. Chem. 8899

Link S, Burda C, Nikoobakht B and El-Sayed M A $2000 \mathrm{~J}$. Phys. Chem. B104 6152

Liu F -K, Chang Y -C, Ko F -H and Chu T -C 2004 Mater. Lett. 58373

Martin C R 1996 Chem. Mater. 81739

Mohamed M B, Ismail K Z, Link S and El-Sayed M A 1998 J. Phys. Chem. B102 9370

Nair A S 2006 Chemistry of halocarbons with bare and protected silver and gold nanoparticles, $\mathrm{Ph} \mathrm{D}$ Thesis, Indian Institute of Technology, Madras

Nair A S and Pradeep T 2003 Curr. Sci. 841560

Nair A S and Pradeep T 2004 Indian Patent No. 51/CHE/2004

Nair A S and Pradeep T 2007 International Patent PCT application no. PCT/IN05/00022

Norberg N S, Dalpian G M, Chelikowsky J R and Gamelin D R 2006 Nano Lett. 62887

Prakash A, McCormick A V and Zachariah M R 2005 Nano Lett. 51357

Rajeev Kumar V R, Samal A K, Sreeprasad T S and Pradeep T 2007 Langmuir 238667

Rao C N R, Kulkarni G U, Thomas P J and Edwards P P 2000 Chem. Soc. Rev. 2827

Rodriguez-Fernandez J, Perez-Juste J, Mulvaney P and LizMarzan L M 2005 J. Phys. Chem. B109 14257 
Salem A K, Searson P C and Leong K W 2003 Nat. Mater. 2 668

Sau T K and Murphy C J 2004 Langmuir 206414

Smith E A and Corn R M 2003 Appl. Spectrosc. 57 320A

Song D K, Lenggoro I W, Hayashi Y, Okuyama K and Kim S S 2005 Langmuir 2110375

Sreeprasad T S, Samal A K and Pradeep T 2007 Langmuir 23 9463

Subramaniam C, Pradeep T and Chakrabarti J 2005 Phys. Rev. Lett. 95164501

Subramaniam C, Pradeep T and Chakrabarti J 2007 J. Phys. Chem. C111 19103

Todd B D and Lynden-Bell R M 1993 Surf. Sci. 281191
Tom R T, Samal A K, Sreeprasad T S and Pradeep T 2007 Langmuir 231320

Tsung C K, Kou X, Shi Q, Zhang J, Yeung M H, Wang J and Stucky G D 2006 J. Am. Chem. Soc. 1285352

Uppenbrink J, Johnston R L and Murrell J N 1994 Surf. Sci. 304223

Wang Z L, Gao R P, Nikoobakht B and El-Sayed M A $2000 \mathrm{~J}$. Phys. Chem. B104 5417

Yu Y Y, Chang S S, Lee C L and Wang C R C 1997 J. Phys. Chem. B101 6661

Zhan B-Z, White MA, Lumsden M, Mueller-Neuhaus J, Robertson K N, Cameron T S and Gharghouri M 2002 Chem. Mater. 143636 